\title{
CHANGES IN COX-2, INOS AND GCL GENE EXPRESSION IN 3T3-L1 PREADIPOCYTES INCUBATED IN MACROPHAGE CONDITIONED MEDIUM
}

\author{
Yoana Kiselova-Kaneva, Oskan Tasinov, Deyana Vankova, Diana Ivanova \\ Laboratory of Nutrigenomics, Functional Foods and Nutraceuticals, \\ Department of Biochemistry, Molecular Medicine and Nutrigenomics, \\ Medical University of Varna
}

\begin{abstract}
PURPOSE: Obesity is recognized as a major risk factor for a number of diseases, including metabolic syndrome and type 2 diabetes mellitus. It is characterized with increased macrophage infiltration in adipose tissue. Macrophages secrete numerous inflammatory mediators and contribute to the increased inflammatory status of adipose tissue which is associated with increased reactive oxygen species generation and oxidative stress. In cell biology conditioned nutrient mediums (CMs) are used to assess the complex impact of the secretory products of a particular cell type on the behaviour of other cells. The aim of the present study was to examine the effect of CM obtained from J744A.1 macrophage cell culture on expression in 3T3-L1 preadipocytes of some enzymes related to antioxidant defense and the inflammatory response.

MATERIAL AND METHODS: mRNA levels of inducible cyclooxygenase (COX-2), inducible NO synthase (iNOS) and glutamate-cysteine-ligase (GCL) were measured. 3T3-L1 cells were treated with increasing CM concentrations (10-30\%) for 24 hours. Changes in gene expression levels were analyzed by real-time qPCR method. Calculations were performed using 2- $\Delta \Delta \mathrm{Ct}$ method.

RESULTS: The higher CM concentration contributed to increased expression levels of the genes examined. Treatment with CM in concentrations of 10, 20 and 30\% resulted in 2.3-, 2.6- and 2.7-fold increase in COX2 expression $(\mathrm{p}<0,01)$, respectively. Both $10 \%$ and $20 \% \mathrm{CM}$ up-regulated iNOS by 1,7 times $(\mathrm{p}<0,001)$. The highest CM concentration of $30 \%$ stimulated the enzyme by two times $(p<0,01)$. GCL $m$ RNA levels were by 2,5 times higher than these of the untreated controls when stimulated with $30 \% \mathrm{CM}(\mathrm{p}<0,05)$.

CONCLUSION: CM from J744A.1 cell culture may induce inflammatory and oxidative stress-related response in 3T3-L1 preadipocytes thus demonstrating a possible link between macrophage infiltration and local inflammation in adipose tissue.
\end{abstract}

Key words: 3T3-L1 preadipocytes, J744A.1 macrophages, conditioned medium, COX-2, iNOS, GCL

Address for correspondence:

Diana Ivanova, PhD

Laboratory of Nutrigenomics, Functional Foods and

Nutraceuticals,

Department of Biochemistry, Molecular Medicine and Nutrigenomics, Medical University of Varna 55 Marin Drinov Street, 9002 Varna, Bulgaria tel: +359-52677075, mobile: $+359-888259786$

e-mail:divanova@mu-varna.bg

Received: February 10, 2013

Accepted: March 22, 2013

\section{INTRODUCTION}

Conditioned medium $(\mathrm{CM})$ is a nutrient medium harvested from cultured cells. It contains metabolites, secretory products (growth factor, cytokines, etc.), and extracellular matrix proteins released into CM by the cultured cells. CMs are used to assess the complex impact of a particular cell type on the behaviour of other cells.

Obesity is recognized as a major risk factor for a number of diseases, including metabolic syndrome 
Yoana Kiselova-Kaneva, Oskan Tasinov, Deyana Vankova et al.

and type 2 diabetes mellitus. It is characterized by increased macrophage infiltration in adipose tissue (12). Adipose tissue macrophages are suspected to be the major source of inflammatory mediators such as TNF- $\alpha$ and IL- 6 that interfere with adipocyte function by increasing inflammatory profile and inhibiting insulin action $(10,14)$. Increased inflammatory status of obese adipose tissue is associated with elevated reactive oxygen species generation and oxidative stress (2).

Effects of macrophages on adipose tissue metabolism have been studied by $\mathrm{CM}$ application. For example, macrophage $\mathrm{CM}$ was found to block insulin action in 3T3-L1 cells (5). CM from macrophages stimulated transcription of proinflammatory genes such as IL-6, IL- $1 \beta$ and MCP-1 in cultured adipocytes $(8,9)$. In the same time, there is a limited evidence of the effect on inflammation-related enzymes iNOS and COX-2. Little is known about the mechanism by which macrophages affect redox balance in adipocytes.

The objective of the present study was to examine the effect of CM obtained from J744A.1 macrophage cell culture on 3T3-L1 preadipocytes by measuring the changes in expression of COX2 and iNOS enzymes related to inflammation and glutamate-cysteine ligase (GCL), an enzyme related to antioxidant defense.

\section{MATERIAL AND METHODS Cell culture}

3T3-L1 and J744A.1 cells were obtained from the American Type Culture Collection (ATCC). Cells were propagated in $75 \mathrm{~cm}^{2}$ flasks at $37^{\circ} \mathrm{C}$ in a humidified chamber with $5 \% \mathrm{CO}_{2}$ atmosphere. Complete nutrient medium comprised phenol of red-containing Dulbecco's Modified Eagle's medium (DMEM, Lonza) with 4,5 g/L glucose, L-glutamine and supplemented with fetal bovine serum (FBS, Sigma-Aldrich) to a $10 \%$ final concentration and penicillin/streptomycin mixture to final concentrations of $100 \mathrm{U} / \mathrm{mL}$ for each of them.

\section{CM collection}

For CM preparation, J744A.1 macrophages were seeded in $75 \mathrm{~cm}^{2}$ flasks at density of 106 cells/ flask in complete medium, which was changed every two days for a period of eight days. Last incubation was for 48 hours in phenol red free medium without any supplements when $\mathrm{CM}$ was collected and frozen at $-80^{\circ} \mathrm{C}$ for further application.

\section{Experimental procedure}

3T3-L1 cells were collected and seeded in 6 well flasks at density of $2 \times 105$ cells/well in complete nutrient medium. After overnight incubation, medium was removed and cells were treated with increasing CM concentration (10\%, 20\% and 30\%) dissolved in phenol red free DMEM (Lonza). No other supplements were used. Cells were incubated for a period of 24 hours prior to RNA extraction.

\section{Gene expression analysis}

Quantitative gene expression analysis was performed using two-step real-time $\mathrm{qPCR}$ as previously described (4). Primer sequences were as follows: mouse $\beta$-actin forward 5'-ACG GCC AGG TCA TCA CTA TTG-3', reverse CAA GAA GGA AGG CTG GAA AAG; mouse COX-2 forward 5'TGA GCA ACT ATT CCA AAC CAG C-3', reverse 5'- GCA CGT AGT CTT CGA TCA CTA TC-3'; mouse iNOS forward 5'- GGC AGC CTG TGA GAC CTT TG-3', reverse 5'- GCA TTG GAA GTG AAG CGT TTC-3`; mouse GCL catalytic subunit forward 5'-AAT GGA GGC GAT GTT CTT GAG-3', reverse 5'-CAG AGG GTC GGA TGG TTG G-3'.

\section{Statistical analysis}

Statistical analyses were performed using Microsoft Excell Office 2007 software. Differences between two groups were analyzed by unpaired twotailed Student's $t$-tests. P value $<0.05$ was considered as significant.

\section{RESULTS AND DISCUSSION}

3T3-L1 preadipocyte cell line is widely used as a model in studies on adipose tissue metabolism and cell culture physiology. J744A.1 is a differentiated macrophage cell line and cultured cells secrete in the nutrition medium IL- $1 \beta$ and other inflammatory cytokines. High IL-1- $\beta$ concentrations could upregulate expression of inflammation-related genes in adipocytes (9).

In the present study, CM collected from J744A.1 macrophages was used to induce inflammatory response in 3T3-L1 preadipocytes and changes in the expression of inflammatory enzymes COX-2 and iNOS were measured to examine inflammatory 
status. Also changes in the expression of an enzyme involved in glutathione metabolism (GCL) were estimated to assess variations in the redox balance of the cells. Most studies relate elevated inflammatory response in cultured adipocytes after incubation in macrophage $\mathrm{CM}$ to up-regulated cytokine gene expression such as IL-6, MCP-1, and TNF- $\alpha$ (9). We established an increased COX-2 and iNOS gene expression after incubation of the predipocytes with increasing concentrations of macrophage CM representing elevated inflammatory cell response (Fig. 1 and Fig. 2).

CM in concentrations of $10 \%, 20 \%$ and $30 \%$ induced 2.3-, 2.6- and 2.7-fold ( $\mathrm{p}<0,01)$ increase in COX-2 mRNA levels, respectively. Both $10 \%$ and $20 \% \mathrm{CM}$ concentrations induced an increased iNOS

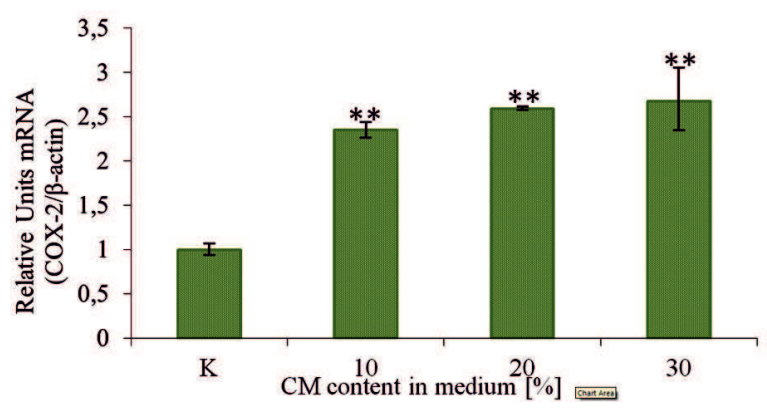

Fig. 1. COX-2 mRNA levels in 3T3-L1 preadipocytes after incubation with macrophage CM. Expression levels are presented in relative units \pm SEM versus $\beta$-actin as endogenous control ${ }^{* *} p<0,01$ versus untreated control

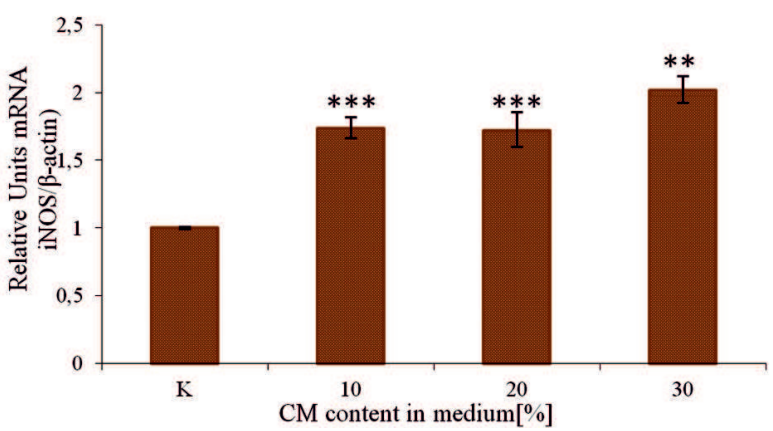

Fig. 2. iNOS mRNA levels in 3T3-L1 preadipocytes after incubation with macrophage CM. Expression levels are presented in relative units \pm SEM versus $\beta$-actin as endogenous control ${ }^{* *} p<0,01$ versus untreated control ${ }^{* * *} p<0,001$ versus untreated control expression by 1,7 times $(\mathrm{p}<0,001)$. The highest $\mathrm{CM}$ concentration (of 30\%) induced iNOS transcription by two times $(\mathrm{p}<0,01)$ (Fig. 2$)$.

COX-2 is the rate-limiting enzyme in production of a range of local mediators involved in various processes, including local inflammation. This enzyme is consistently induced by inflammatory stimuli such as LPS, IL- $1 \alpha / \beta$ and TNF- $\alpha$ and is a key executor of inflammation in many cell types, including adipocytes $(3,7)$. Our data about elevated COX-2 expression after treatment with macrophage $\mathrm{CM}$ indicate another possible link between macrophage infiltration and adipose tissue inflammation.

iNOS is the inducible isoform of the enzyme catalyzing $\mathrm{NO}$ synthesis in response to inflammatory stimuli. iNOS is expressed in adipose tissue (1) and in 3T3-L1 cells, iNOS is induced by lipopolysaccharides and proinfammatory cytokines including TNF- $a$ and interferon- $\gamma$ (11). Elevated iNOS expression is observed in macrophages isolated from obese adipose tissue $(5,13)$. Our results show that macrophagederived CM may induce iNOS expression, which in addition to up-regulated COX-2 expression represents a provoked inflammatory response in 3T3-L1 cells.

There was a stimulated antioxidant defense in the 3T3-L1 preadipocytes after incubation in macrophage CM. GCL mRNA levels in cells treated with $30 \% \mathrm{CM}$ are by 2,5 times $(\mathrm{p}<0,05)$ higher than that of the untreated controls (Fig. 3). It is accepted that inflammation is associated with impaired redox

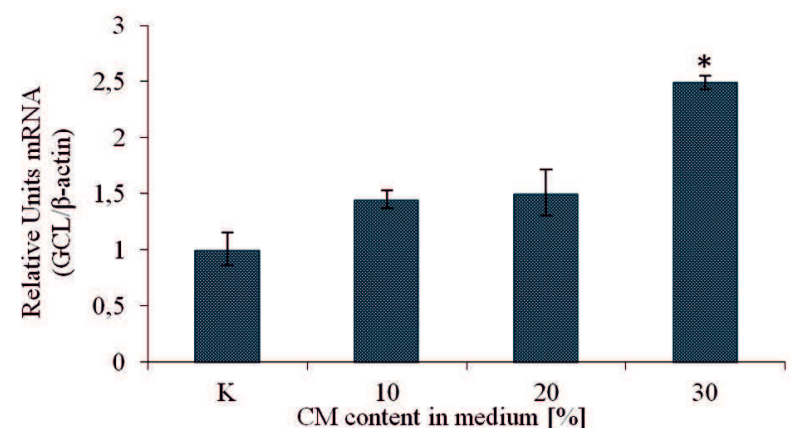

Fig. 3. GCL mRNA levels in 3T3-L1 preadipocytes after incubation with macrophage CM. Expression levels are presented in relative units \pm SEM versus $\beta$-actin as endogenous control ${ }^{*} p<0,05$ versus untreated control 
Yoana Kiselova-Kaneva, Oskan Tasinov, Deyana Vankova et al.

balance (2). GCL is the first and rate-limiting enzyme in glutathione biosynthesis. Up-regulated GCL expression to ensure GSH elevation under conditions of inflammatory and oxidative stimulation seems reasonable, as GCL activity is considered especially important for maintaining GSH levels (6) to provide relevant antioxidant defense.

\section{CONCLUSION}

This study demonstrates that CM from J744A.1 macrophages may induce inflammatory and antioxidant response in cultured 3T3-L1 preadipocytes proved by the elevated COX-2, iNOS and GCL transcription.

\section{ACKNOWLEDGEMENTS}

The study was supported by COST Action BM0602.

\section{REFERENCES}

1. Engeli, S., J. Janke, K. Gorzelniak, J. Böhnke, N. Ghose, C. Lindschau, et al. Regulation of the nitric oxide system in human adipose tissue.- J. Lipid Res., 45, 2004, No 9, 1640-1648.

2. Fernández-Sánchez, A., E. Madrigal-Santillán, M. Bautista, J. Esquivel-Soto, A. Morales-González, C. Esquivel-Chirino, et al. Inflammation, oxidative stress, and obesity.- Int. J. Mol. Sci., 12, 2011, No 5, 3117-3132.

3. Herschman, H. R. Prostaglandin synthase 2.Biochim. Biophys. Acta, 1299, 1996, No 1, 125-140.

4. Kiselova-Kaneva, Y., O. Tasinov, D. Vankova, D. Ivanova. Ethanol induces IL- 6 and TNF- $\alpha$ cytokine and iNOS and COX-2 enzyme gene expression in 3T3-L1 preadipocytes.- Scr. Sci. Med., 44, 2012, No 2, 31-35.

5. Lumeng, C. N., J. L. Bodzin, A. R. Saltiel. Obesity induces a phenotypic switch in adipose tissue macrophage polarization.- J. Clin. Invest., 117, 2007, No 1, 175-184.
6. Morales, A., C. Garcia-Ruiz, M. Miranda, M. Mari, A. Colell, E. Ardite, et al. Tumor necrosis factor increases hepatocellular glutathione by transcriptional regulation of the heavy subunit chain of gamma-glutamylcysteine synthetase.- J. Biol. Chem., 272, 1997, No 48, 30371-30379.

7. Morita, I. Distinct functions of COX-1 and COX-2.Prostaglandins Other Lipid Mediat., 68-69, 2002, 165-175.

8. O’Hara, A., F. Lim, D. J. Mazzatti, P. Trayhurn. Stimulation of inflammatory gene expression in human preadipocytes by macrophage-conditioned medium: upregulation of IL- 6 production by macrophage-derived IL-1ß.- Mol. Cell. Endocrinol., 349, 2012, No 2, 239-247.

9. Permana, P. A., C, Menge, P. D Reaven. Macrophage-secreted factors induce adipocyte inflammation and insulin resistance.- Biochem. Biophys. Res. Commun., 341, 2006, No 2, 507-514.

10. Suganami, T., Y. Ogawa. Adipose tissue macrophages: their role in adipose tissue remodeling.- J. Leukoc. Biol., 88, 2010, No 1, 33-39.

11. Unno, Y., T. Akuta, Y. Sakamoto, S. Horiuchi, T. Akaike. Nitric oxide-induced downregulation of leptin production by 3T3-L1 adipocytes.- Nitric Oxide, 15, 2006, No 2, 125-132.

12. Wellen, K. E., G. S. Hotamisligil. Obesity-induced inflammatory changes in adipose tissue.- J. Clin. Invest., 112, 2003, No 12, 1785-1788.

13. Weisberg, S. P., D. McCann, M. Desai, M. Rosenbaum, R. L. Leibel, A. W. Ferrante Jr. Obesity is associated with macrophage accumulation in adipose tissue.- J. Clin. Invest., 112, 2003, No 12, 1796-1808.

14. Zeyda, M., T. M. Stulnig. Adipose tissue macrophages.- Immunol. Lett., 112, 2007, No 2, 61-67. 